\title{
Haptic feedback perception and learning with cable-driven guidance in exosuit teleoperation of a simulated drone
}

\author{
Carine Rognon, Student Member, IEEE, Vivek Ramachandran, Student Member, IEEE, \\ Amy $\mathrm{R}$ Wu, Member, IEEE, Auke J ljspeert, Senior Member, IEEE, \\ and Dario Floreano, Senior Member, IEEE
}

\begin{abstract}
Robotics teleoperation enables human operators to control the movements of distally located robots. The development of new wearable interfaces as alternatives to hand-held controllers has created new modalities of control, which are more intuitive to use. Nevertheless, such interfaces also require a period of adjustment before operators can carry out their tasks proficiently. In several fields of human-machine interaction, haptic guidance has proven to be an effective training tool for enhancing user performance. This work presents the results of psychophysical and motor learning studies that were carried out with human participant to assess the effect of cable-driven haptic guidance for a task involving aerial robotic teleoperation. The guidance system was integrated into an exosuit, called the Flyjacket, that was developed to control drones with torso movements. Results for the Just Noticeable Difference (JND) and from the Stevens Power Law suggest that the perception of force on the users' torso scales linearly with the amplitude of the force exerted through the cables and the perceived force is close to the magnitude of the stimulus. Motor learning studies reveal that this form of haptic guidance improves user performance in training, but this improvement is not retained when participants are evaluated without guidance.
\end{abstract}

Index Terms-Haptics and Haptic Interfaces, Physical Human-Robot Interaction, Human Performance Augmentation, Telerobotics

\section{INTRODUCTION}

$\mathrm{D}$ RONES have become an ever-present feature in a multitude of robotic applications, ranging from crop surveillance and mapping for agriculture to package delivery for supply-chain logistics to transmission line monitoring for large-scale infrastructure maintenance. Indeed, aerial robotics has proven to be a boon for humans, assisting in risk-prone tasks such as search-and-rescue and post-disaster inspection thereby extending intrinsic human perception and providing them with three-dimensional mobility [1]. However, mastering the control of drones for precise tasks requires hours of training, and in most cases, professionals in the aforementioned sectors cannot afford to expend resources towards this endeavour [2].

In order to increase the accessibility of drone technology to a wider population, more intuitive and natural controllers are needed. Previous work provided haptic feedback through a hand-held remote controller to give additional

- C. Rognon is with the Laboratory of Intelligent Systems, Ecole Polytechnique Federale de Lausanne, 1015 Lausanne, Switzerland. E-mail: carine.rognon@epfl.ch

- $\quad V$. Ramachandran is with the Laboratory of Intelligent Systems, Ecole Polytechnique Federale de Lausanne, 1015 Lausanne, Switzerland. E-mail: vivek.ramachandran@epfl.ch

- A. R. Wu is with the Biorobotics Laboratory, Ecole Polytechnique Federale de Lausanne, 1015 Lausanne, Switzerland. E-mail: amy.wu@epfl.ch

- A. Ijspeert is with the Biorobotics Laboratory, Ecole Polytechnique Federale de Lausanne, 1015 Lausanne, Switzerland. E-mail: auke.ijspeert@epfl.ch

- D. Floreano is with the Laboratory of Intelligent Systems, Ecole Polytechnique Federale de Lausanne, 1015 Lausanne, Switzerland.

E-mail: dario.floreano@epfl.ch

Manuscript received November 15, 2018 information to the pilot about the state of system, such as the distance to obstacles [4], [5], the drone position (Motion Pilot, Ecublens, Switzerland), or drone velocity [6], [7]. However, the provision of haptic feedback alone is insufficient to overcome the inherent problem of non-intuitiveness that comes with using hand-held remote controllers. A recent study identified natural upper body gestures used by naive users to control drones [8]. The gesture pattern consist of torso movements to control the drone and makes the task easier for naive users in comparison to the use of traditional remote controllers. In order to record these upper body movements and transmit commands to the drone, we developed a soft upper body exoskeleton, called the FlyJacket [9]. The control was implemented by a linear mapping between the orientations of the human torso and of the drone. Bending the torso in the sagittal plane forward and backward made the drone pitch down and up respectively. Bending the torso in the frontal plane made the drone roll in the corresponding directions. With human participant experiments, we found that the flight precision increased when participants used a flight style with their arms spread out. However, prolonged maintenance of this position induced fatigue. Therefore, the FlyJacket was equipped with removable and passive arm supports that prevented arm fatigue. Results of flights with participants showed that using intuitive upper body movements recorded with the FlyJacket could make the interaction with the drone more intuitive and natural than when using a remote controller. In addition, the variance of the performance over participants was significantly smaller when using intuitive body gesture to control a drone, i.e. all the participants that used the 
A

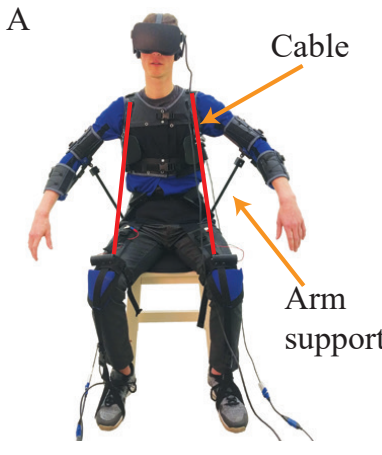

B

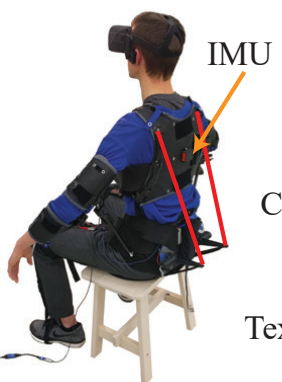

C

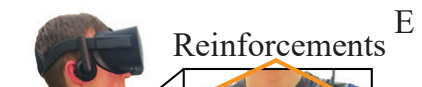

$\mathrm{E}$
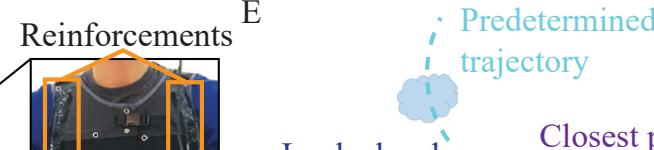

Look ahead 'Closest point

$1 \ldots$

point on the trajectory

Attractive force

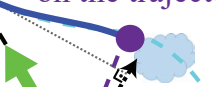

$(8)$ padding

(lile

band

Drone

Waypoint

Textile band

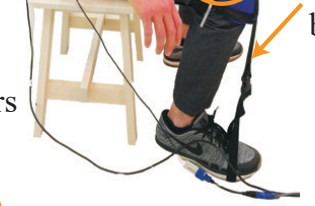

.

Fig. 1. Experimental setup of the FlyJacket with the haptic feedback cable-driven actuation. Figure adapted from [3]. Cables are highlighted in red. (A) Front view of a participant wearing the FlyJacket and the head mounted display. (B) Back view in perspective showing the IMU. (C) Side view highlighting the motors for actuation and an inset showing the thermoplastic reinforcements. (D) Zoomed in the back motor showing the cantilever mechanism. (E) 2D schema of the drone trajectory and the haptic guidance. The attractive force is quadratically related to the distance between the drone and a look ahead point on the predetermined trajectory. (F) Image of the flight environment highlighting the waypoints with an inset showing how a waypoint is seen by the participant when they fly towards it.

FlyJacket could reach a good performance level while only skilled participants performed well when using the remote controller.

Additional information about the body position in space could be given to the user in order to further improve the flight precision. For complex tasks such as flying, adding haptic feedback led to a faster reaction time than solely relying on visual and auditory feedback [10], [11]. Moreover, haptic feedback has been shown to accelerate the learning of a task in many fields such as surgery [12], rehabilitation [13], and sports [14], [15]. Rendering flight information using haptic feedback on the user's torso could provide a collocated feedback that is more natural and easier to process [16] and help the user to learn at as faster rate.

For this purpose, a cable-driven device has been added in the FlyJacket in order to give haptic guidance in the form of kinesthetic feedback to the users torso [3] (see Fig. 1). With this device, forces can be applied to the torso to correct user position in order to reach a target or to avoid collision with obstacles. The device is made of four electrical motors (DC22S, gear ratio 6.6:1, Maxon Motor, Switzerland) pulling on cables (Dyneema $0.4 \mathrm{~mm}$, Spiderwire, SC, USA, displayed in red in Fig. 1) attached to the upper part of the torso. Each motor of the FlyJackets haptic system can produce up to 30 $\mathrm{N}$ of force. This corresponds to a torque of approximately $20 \mathrm{Nm}$ for a $175 \mathrm{~cm}$ tall user when both motors of one body side are pulling together. The range of force produced by the motors was chosen to be strong enough to apply a torque on the user's torso to overcome the passive torso stiffness $(10 \mathrm{Nm})$ but smaller than the maximum torque the human can produce $(150 \mathrm{Nm})$ so the user could resist the applied force. For the front motors, one motor is placed on the distal part of each leg. To prevent them from sliding along the legs when pulling on the cables, they are maintained by a nonelastic textile band attached at its extremity to the users feet by the mean of a loop (see Fig. $1 \mathrm{C}$ ). The two back motors are located on each side of the lower back. The back cables are passing from the motor through cantilevers (made of 3D printed Acrylonitrite Butadiene Styrene (ABS)) which create a lever arm to induce forces that pull the user backward, instead of downward (see Fig. 1 C and D). To hinder the motor from moving up when pulling on the cables, they are fixed on a rigid plate and the extremities of the cantilever tips are also attached with a non-elastic bands passing on the back of the tight with the user being sited on them. As the cables are attached on the torso part made of leather, reinforcements made of polymorph thermoplastic (Thermoworx Ltd, Ayrshire, Scotland, UK) have been inserted to stiffen the structure in order to prevent force losses and transmission delays (see inset of Fig. 1 C). Padding on the knee and lower back has been added to avoid user discomfort due to the force routing. The four motors are independently controlled by four transistors activated through a control board (Arduino Uno, Arduino, Italy). Thanks to their low gear ratio, motors are back-drivable. Therefore, they are only activated when a corrective force is required to pull on the cables. With the antagonistic configuration and four independently controlled motors, the applied forces can bend the user in both the sagittal and frontal planes and therefore can correct the torso's position in roll, pitch or a combination of both.

In a previous user study [3], this setup have been tested 
in a short flight task of following waypoints in the sky (Fig. 1 $\mathrm{E}$ and $\mathrm{F}$ ). The aims of this past work were to determine the effect of haptic guidance on flight performance and the best force profile to guide the user. We found that a quadratically-shaped haptic guidance increased user performance in comparison to flying without any guidance. Participants also subjectively reported that haptic guidance while flying was useful. However, the long term effects of the guidance, as well as participants perception of the force applied, remained unclear. If the user could learn to fly better with haptic guidance, such a device could be used for training, and the guidance could be removed once the user reached a sufficient level of performance. In order to design the training task and the type of guidance provided during this period, it is important to understand how users perceive this type of haptic feedback, in particular the minimal force difference they can discern and the force magnitude they perceive on the front and on the back of their torso. Moreover, it is imperative to determine the threshold of human force perception as previous work has shown that the provision of sub-threshold force feedback can be detrimental to user performance in haptic-enabled virtual environments [17].

The study of human force perception was intensively carried out by G.T. Fechner and E.H. Weber [18]. They developed the Just Noticeable Difference (JND) test which study the difference required by human to perceive changes in stimuli [19]. S. S. Stevens extended this work and demonstrated that stimuli perceptions do not follow a logarithmic law but a power law [18], [20], [21]. Stevens' work on tactile sensations through vibrations demonstrated a linear correspondence between a stimulus and the perceived magnitude [22]. It is uncertain if a similar linear relationship holds for kinesthetic stimulus on the torso.

In this article, we investigated what the most effective way would be to transmit the information to the user and the retention of this information with the same cable-driven device. To address these questions, we conducted two studies. First, psychophysical experiments were performed to determine how the user perceived the force applied to their body through determining the Just Noticeable Difference [19] and the coefficients for the Stevens' power law [20]. Second, flight experiments were conducted to determine the influence of the haptic guidance on user performance for an extensive flight task and the level of short term information retention when the haptic guidance was removed. All participants to these two studies provided written informed consent prior to the study in accordance with the EPFL Institutional Review Board procedures.

\section{Haptic Feedback Perception}

The first phase of research investigated the sensitivity of FlyJacket users to a given force to understand how applied forces are perceived and to improve the control of the drone. It is therefore important to measure the minimal force amplitude the user can perceive and their ability to discern differences in amplitude. In this phase, two sets of tests were conducted on 10 participants. The first set investigated the Just Noticeable Difference (JND), which is the minimal force difference the user can perceive at different force

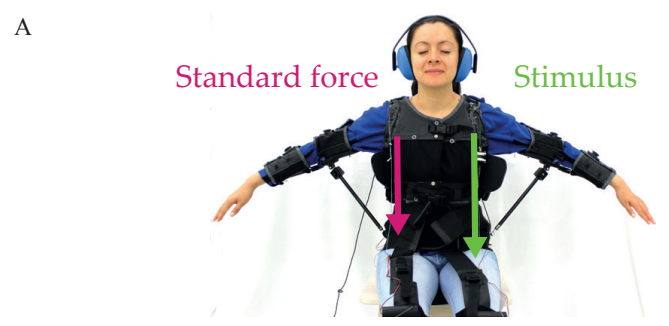

B
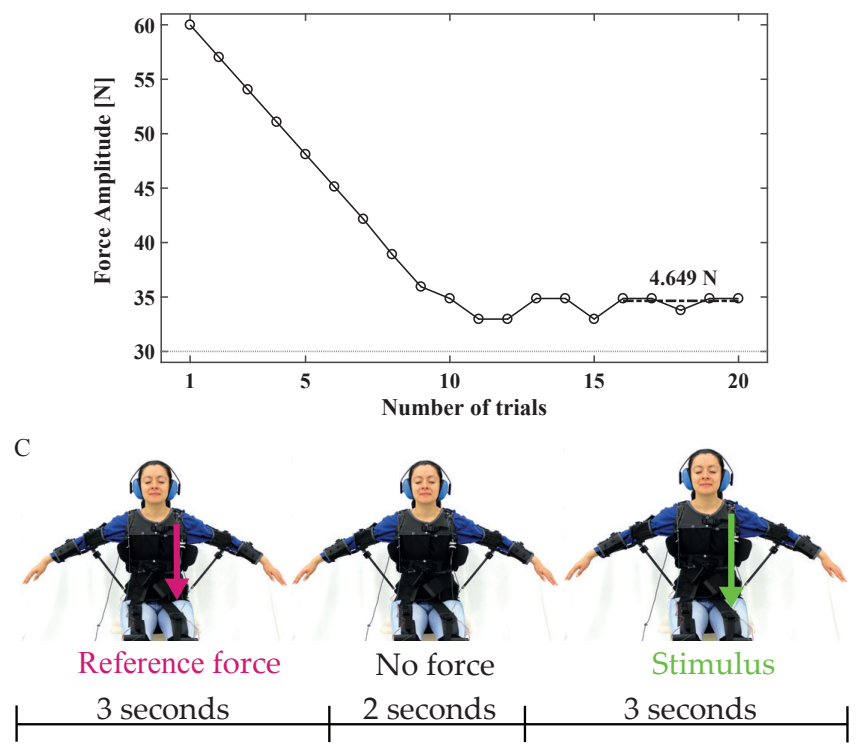

Fig. 2. Haptic feedback perception experimental flow. (A) Experimental setup and schema of the application of the force for the Just Noticeable Difference experiment with the standard force (pink) and the stimulus (green). (B) Exemplar results of the staircase method from one participant using the standard force of $30 \mathrm{~N}$ on the front torso. The dashed black line represents the average over the last five trials (C) Testing procedure for the Stevens power law experiment with a schema of the reference force (pink) and the stimulus (green), here applied on the left side, and timing. The reference force and the stimulus were applied on the same side, alternatively between right and left.

amplitudes. The second set aimed at determining whether perception of haptic feedback to the torso by motor-driven cables followed a linear law between a given stimulus and the participant perception, based on the Stevens' power law.

\subsection{Description of the Experiment}

These two studies were performed on healthy adult participants $(\mathrm{N}=10$, five males, five females, age $23.1 \pm 4.46$ years; mean $\pm \mathrm{SD}$ ). The participants sat on a stool, wearing the FlyJacket with the arm supports and earmuffs to block external noises, such as from the motors (see Fig. 2 A). Participants were asked to close their eyes during the trials. The haptic feedback perception tests measure the force perception on static limbs. Therefore, participants were instructed to stay in an upright position and resist the force applied to their torso (i.e. by keeping their torso aligned with gravity) during all the trials.

The first experiment determined the Just Noticeable Difference - the minimal force difference a participant can perceive for various force amplitude - for four standard force amplitudes $(5,10,20$ and $30 \mathrm{~N})$. The standard force 
was applied on one side of the torso, and on the other side, a proposed stimulus was given simultaneously (see Fig. 2 A). The standard force and the proposed stimulus were applied simultaneously on either side of the upper torso for three seconds. The proposed stimulus amplitude started at twice the amplitude of the standard force. After the standard force and the proposed stimulus were applied, the participant then verbally stated on which side he or she felt the stronger force. Depending on the $s^{\prime}$ answer, the amplitude of the proposed stimulus was adjusted by a step corresponded to $10 \%$ of the standard force amplitude going down in a staircase method "two up, one down" for 20 trials for each standard force (see Fig. 2 B) [23]. If the answer was correct, the proposed stimulus amplitude was reduced by $10 \%$ of the standard force for the 10 first trials. For trials 11 to 20, the participant needed to give two successive correct answers for the proposed force to be reduced. The JND of each of the standard force amplitudes was calculated as the average force of the amplitudes from the last five trials [24]. Averaging among the two to eight last trials did not influence the results. The forces were applied pseudo-randomly on the right and the left of the torso to ensure an equal number of pulls of the proposed stimulus on both sides. This experimental procedure was performed for first the front and then the back of the torso. A linear fit of the JND data was performed using polyfit function in MATLAB (The MathWorks, Inc., Massachusetts, USA) and the coefficient of determination was computed using the rsquare function.

The second experiment aimed at understanding the relationship between a given stimulus and what the user perceives. The objective was to find the coefficients of the Stevens' power law ( $a$ in 1) to determine if receiving kinesthetic feedback with the FlyJacket followed a linear progression over the proposed amplitudes and therefore matched with results previously found for vibrations [22]. For this test, the experimental setup remained the same as for the JND experiment. However, this time the participants received first a reference force of $15 \mathrm{~N}$ randomly on either the left or the right of their torso and then, after a two seconds break, a stimulus at the same place (see Fig. $2 \mathrm{C}$ ). The proposed stimuli $S$ were 5, 10, 15, 20, 25 and $30 \mathrm{~N}$. The reference force was applied for 3 seconds, followed by a 2 second break, and then the stimulus was applied for 3 seconds. The participant then verbally stated their estimate of the perceived force intensity $P$. They were told that the stimulus force could range from $0 \mathrm{~N}$ to $30 \mathrm{~N}$, inclusive. Each stimulus was presented three times for a total of 18 trials. This experiment was performed both for the front and the back of the torso.

A linear fit for each in the log space was performed. The exponent $a$ of the Stevens power law (1) was calculated as the slope of a linear fit of the logarithm of participant data (2) [19]. The multiplier $\lambda$ was calculated as the exponential of the $y$-intercept of the linear fitting in the logarithm scale.

$$
\begin{gathered}
P(s)=\lambda S^{a} \\
\log P(S)=a \log S+\log \lambda
\end{gathered}
$$

The significance of fit parameters $a$ and $\lambda$ were tested using one-sample t-test. The detection threshold was computed as the $y$-intercept $(\log \lambda)$ of the linear interpolation for the front and the back [25].

\subsection{Results for the Just Noticeable Difference}

The results of the JND experiment showed a linear trend of the perception over the standard force tested $(5,10,20,30 \mathrm{~N})$ for both the front and back of the torso (Fig. 3). Results for the front and the back are comparable, and both are strongly correlated with a linear law. From a fit to all s' data, the coefficient of determination of $R^{2}=0.765$ for the front and $R^{2}=0.991$ for the back of the torso. If the data point from one participant at standard force amplitude of $20 \mathrm{~N}$ and JND of $9.62 \mathrm{~N}$ (see red circle sign in Fig. 3) is considered as an outlier - as its JND is greater than the mean of all $\mathrm{s}$ $(3.03 \mathrm{~N})$ plus twice the standard deviation $(0.85 \mathrm{~N})-$ and is removed from the regression, the $R^{2}$ of the front becomes $R^{2}=0.916$. The extrapolation of the linear fit for each to the $y$-intercept predicts that the detection threshold should be $0.63 \mathrm{~N} \pm 0.70$ (mean $\pm \mathrm{SD}$ ) for the front and $0.81 \mathrm{~N} \pm 0.81$ for the back. However, further tests should be performed to confirm this predicted threshold difference between front and back. Following the linear law, the JND for any standard amplitude can be predicted as this threshold plus $10 \%$ of the standard force, SF (the slope of the linear fitting is $0.11 \pm$ 0.08 for the front and $0.09 \pm 0.060$ for the back of the torso). This linear fitting over all the participants has a significant trend $(P<0.05)$ for both the front $(P=0.0019)$ and the back $(P=0.0012)$. With the exception of the $5 \mathrm{~N}$ and $10 \mathrm{~N}$ standard force applied to the back, the standard deviation among participants was small $(6 \%$ of the standard force amplitude). For the two exceptions, the standard deviation across participants was approximately $20 \%$ of the standard force amplitude.

\subsection{Results for Stevens' Power Law}

The results of the Stevens' power law experiment (Fig. 4) show that participants' magnitude perception for proposed stimulus followed a linear law for stimuli applied to the front and the back of the torso. The computed fits for both the front and the back of the torso demonstrated that the perceived magnitudes follow a linear law with Stevens' exponents close to one $\left(a_{\text {front }}=0.97 \pm 0.24\right.$ and $a_{\text {back }}=1.16$ \pm 0.36 , mean $\pm \mathrm{SD}$ ). The correlation with a linear law is strong as the coefficient of determinations of the linear fit are close to one $\left(R^{2}=1.000\right.$ for the front and $R^{2}=0.999$ for the back). However, the multiplier $\lambda$ doubles between the front $\left(\lambda_{\text {front }}=1.03\right)$ and the back $\left(\lambda_{\text {back }}=0.54\right)$ of the torso. Therefore, for the front, users perceived the increase in force linearly and at the same amplitude as intended. In contrast, the measured slope was steeper for the back than the front, with the two lines crossing at $27 \mathrm{~N}$. Therefore, users perceived a lower force for lower stimuli (less than $27 \mathrm{~N}$ ) and a higher perceived force for high stimuli (greater than $27 \mathrm{~N}$ ) on the back.

\subsection{Discussion}

Our perception studies demonstrate that the information transmitted to the user's torso by the haptic force feedback 


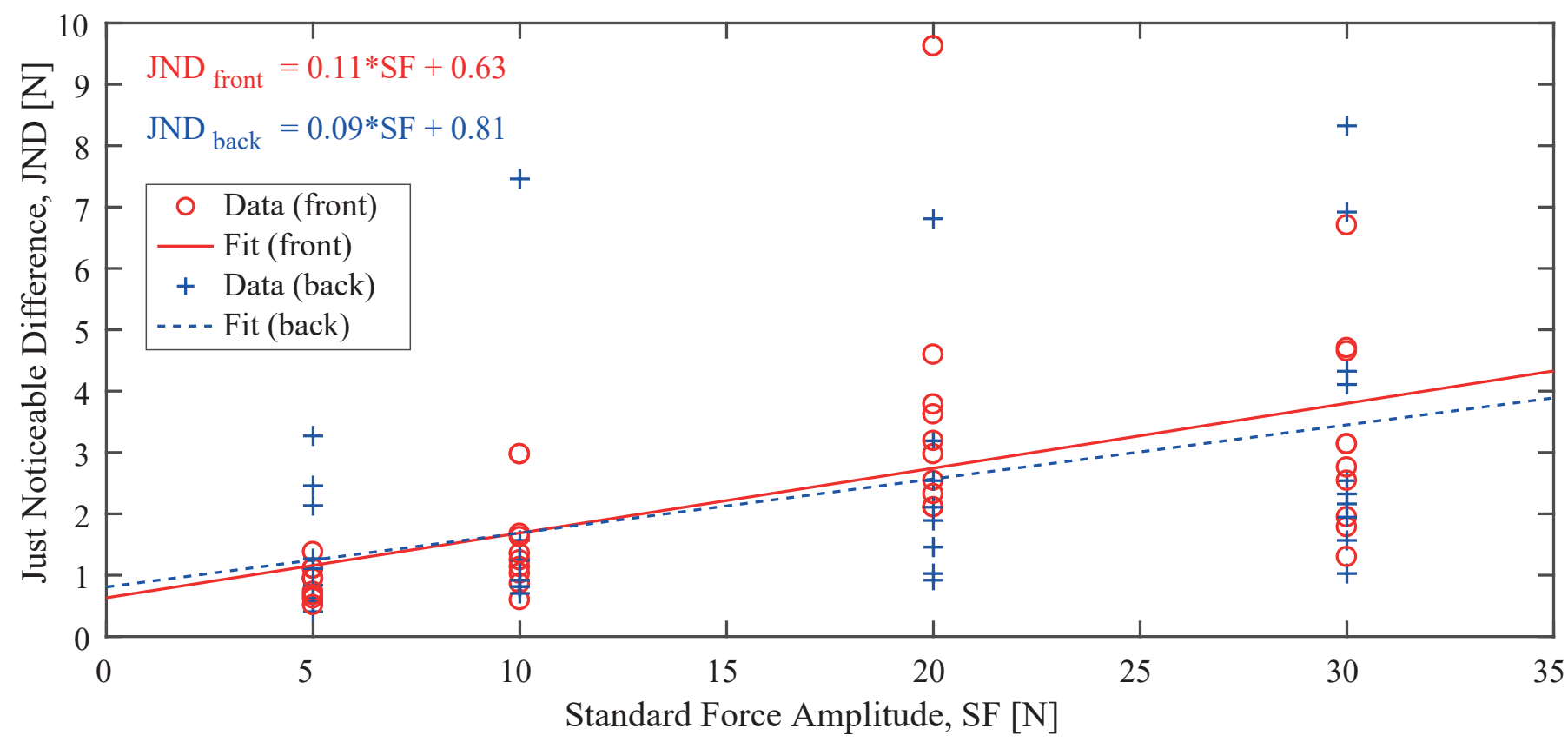

Fig. 3. Just Noticeable Difference as a function of Standard Force Amplitude ( $N=10)$. All participants' data for each standard force amplitude are presented for the front of the torso (red circles) and for the back (blue crosses). The mean of participants' linear fit of the data are shown for the front (solid line) and for the back (dashed line) of the torso.

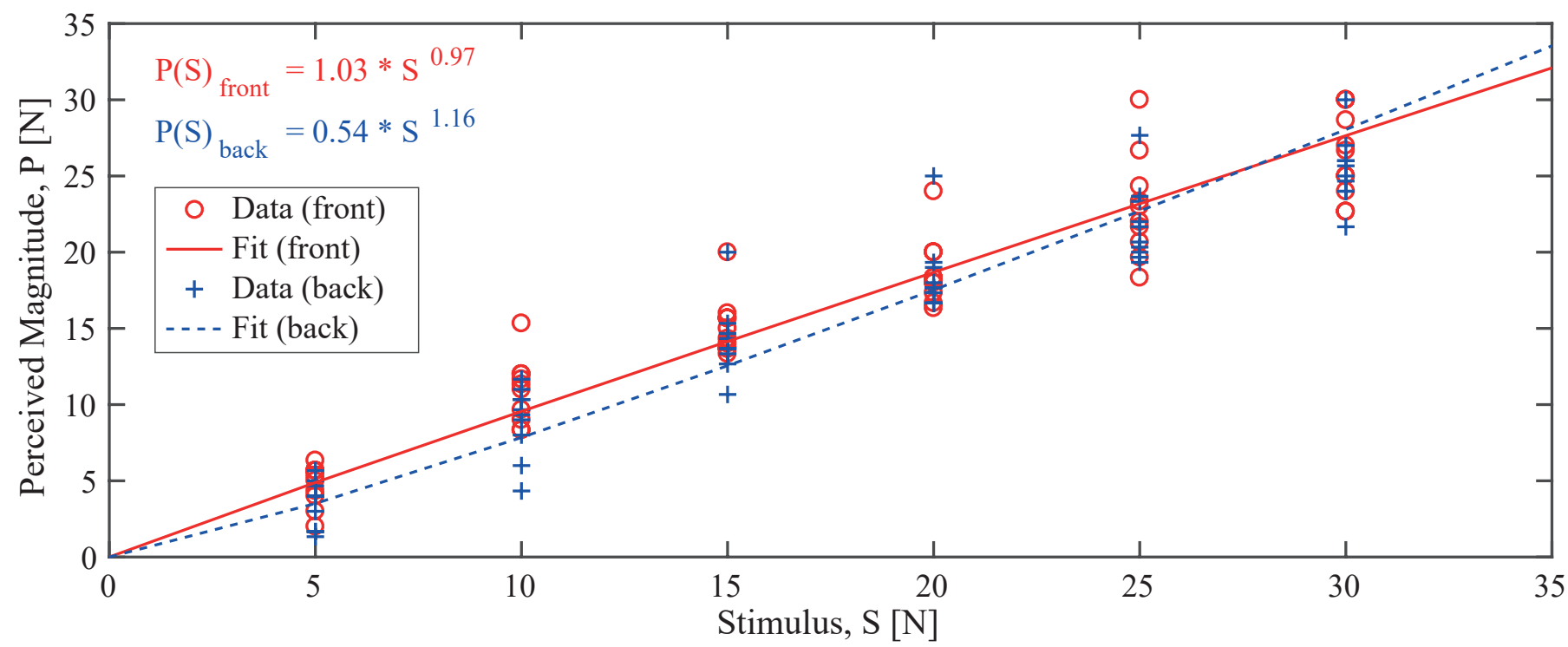

Fig. 4. Perceived magnitude as a function of stimulus from the Stevens' power law experiments $(\mathrm{N}=10)$. All participants' data for each stimulus are presented for the front of the torso (red circles) and for the back (blue crosses). The computed Stevens power equations obtained from the average of $s$ ' fit parameters are shown for the front (solid line) and for the back (dashed line).

will be perceived as intended by the command. The kinesthetic feedback perception also follows a linear tendency for both the JND and the perceived magnitude, which corroborates with similar research on vibrotactile feedback [22], [24], [25]. The JND results show that the minimum force difference a user of the FlyJacket can perceive scales linearly with the amplitude of the given forces. Users will not perceive differences in force lower than $10 \%$ of the stimulus. Therefore, force feedback indications should have a difference in amplitude larger than this threshold. The perceptions for the front and the back of the torso are comparable with a slightly higher detection threshold for the back. The perception of force on the back of the torso is also more variable among $\mathrm{s}$ at lower amplitudes than greater ones. The results of the experiment to determine the coefficients of the Stevens' power law showed that users perceive forces at an intensity similar to the given stimulus, nevertheless, similarly to the JND results, at a slightly lower intensity for the back than the front.

The differences between front and back for both perception experiments could be due to a difference in sensitivity between the front and the back of the torso [26]. Another 
possibility is that the lever arm of the back cables is smaller than for the front cable, resulting in a smaller applied torque for the same force stimulus. However, it is unclear how much influence this torque difference has on perception.

We used the results of these experiments to validate the parameters of the quadratically-shaped force guidance curve (see (3), derived from [3]), that will be used in the flight task. The force guidance curve gives a weak force when the error is small and a strong force when the error is large and is perceived as designed according to the results of the perception experiments. The perception of the minimal force difference is $10 \%$ of the stimulus. The slope of the quadratic term was set at $30 \%$. Therefore, the current guidance force is compelling enough to affect the user, and its changes over time can be perceived by the user. The minimal force threshold that participants could feel was approximately $0.7 \mathrm{~N}$, which is the force they would receive with an error of $2.2 \mathrm{~m}$. This corresponds to approximately the half of the mean RMS error over all participants that took part of a previous flight experiment [3]. Both the JND experiment and the Stevens' power law experiment showed no significant difference in perception between the front and the back of the torso. Therefore, the command can be set identically for the front and the back motors.

As the experimental results of the perception experiment justify the parameters of the quadratically-shaped curve, which was found to have improved the performance during a short flight task, we retained the same parameters for our longer flight task on training.

\section{Performance Improvement During a FLIGHT TASK}

In a previous study [3], we demonstrated that having haptic guidance, by correcting the torso position when flying, increases the user's performance in comparison to flying without any guidance. The best type of force profile was found to be quadratically shaped as it provided a weak guidance when the error was small $(<4$ meters; for reference, the wingspan of the drone is 0.96 meter) and a strong guidance when the error was large ( $>10$ meters). Having a haptic guidance was also valued by participants. With this study, we sought to determine how haptic guidance influenced performance over an extended period of time (9 minutes of flight instead of 2.5 minutes for the previous study) and if the user retained the control skills when the haptic guidance was removed. Results would determine if the training time could be reduced using haptic guidance, what the appropriate amount of training required would be, and whether the user retained the skill acquired with the haptic guidance when the guidance was removed. To evaluate these questions, a user study was performed on participants in which they were asked to perform a flight task of following a trajectory composed of waypoints (wp) in the sky (see Fig. $1 \mathrm{E}$ and F). This task was similar as our previous, shorter flight time study [3].

\subsection{Description of the Experiment}

In total, 27 participants took part in this study, out of which data from 20 participants were used (12 males, 8 females, age $25.85 \pm 4.17$ years; mean \pm SD). Of the 20 participants, eleven received haptic guidance during the training task and nine flew without any haptic guidance. Nine of these 20 participants also took part in the haptic feedback perception experiment. Of the seven participants excluded from this study, five participants could not finish the experiment due to feelings of dizziness, one participant had to stop because of technical problems with sensors, and one participant stopped the experiment after the training as he was unable to control the drone. The standard deviation of the performance of this last excluded participant over the 150 waypoints of the training task was $24.40 \mathrm{~m}$, which is six times greater than the mean of the standard deviation of the other participants flying without feedback $(4.55 \mathrm{~m})$.

Participants began the study by completing a questionnaire about their physical traits (height and weight), handedness (left or right), visual acuity, hours of physical activities, and experience in playing video games and piloting remotely an aircraft. Then, the participants wore the FlyJacket with the passive arm support and sat on a stool (see Fig. 1). They wore virtual reality goggles (Oculus Rift, Facebook, CA, USA), which provided the visual feedback of the simulated environment. During this experiment, participants had to control a fixed-wing drone in a simulator developed in Unity3D (Unity Technologies, CA, USA) using upper body movements described in [9]. The movements to control the drone were demonstrated to them by the experimenter, and the experimental protocol was explained (see Fig. 5).

Participants began with a short familiarization of the flight movements and simulator without any haptic guidance. This familiarization task was composed of two parts. At first, they were asked to follow an arrow in the sky pointing to the right, left, up and down. This sequence was presented to them twice in order to have them practicing all the movements to control the drone. The second part was one and a half minutes of free flight in a reconstructed virtual environment of the EPFL campus. The goal was for them to practice the movements and accustom themselves to the control of the drone.

After this short familiarization phase, participants started the next phase of following waypoints, symbolized by small white clouds of diameter one meter, forming a trajectory in the sky (see Fig. $1 \mathrm{D}$ and E). The waypoints were spaced apart by approximately 30 meters. Experimenters explained to the participants that the distance between the drone and the center of each cloud was recorded and that their goal was to minimize their error over all the waypoints. The order of the maneuvers to reach the waypoints (up, down, left, right) was randomized between trials, but the number of maneuvers was always the same between participants. Participants had to perform three types of tasks named "Baseline", "Training", and "Evaluation" (see Fig. 5). Their first task "Baseline" was to fly through 26 waypoints, which took approximately a minute and a half, without any haptic guidance. This task was used to calculate their baseline performance. Next, the participants completed a questionnaire to rate on a Likert scale (from 1 to 7 ) how strongly they agreed with the following statements concerning their subjective control ability and flight sensations (1 being strongly disagree and 7 strongly agree): 
Familiarization Baseline Training Evaluation

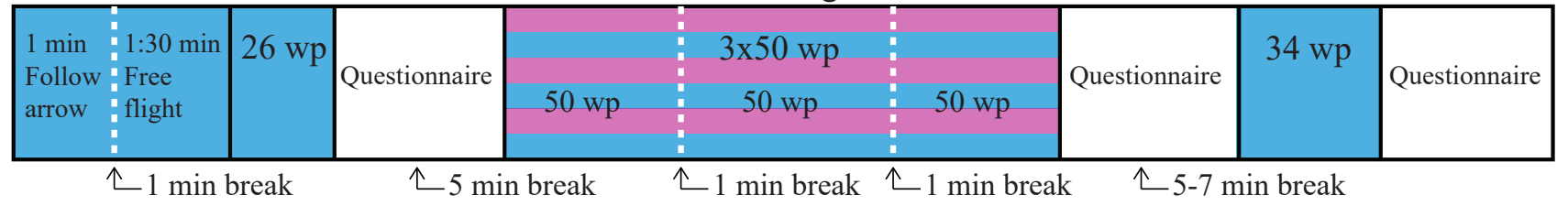

Fig. 5. Flight experiment protocol, indicating the tasks performed without guidance (blue) and with guidance (magenta). During the training, eleven participants flew with guidance and nine participants flew without guidance. wp: waypoint.

1) "I felt as if I was flying,"

2) "I had the feeling of controlling the flight trajectory,"

3) "The training was clear and sufficient,"

4) "I enjoyed the experiment,"

5) "I felt dizzy,"

6) "I felt some physical discomfort,"

7) "The setup was comfortable,"

8) "My movement did not feel constrained during the flight."

The second part was the training phase. It was composed of three sessions of 50 waypoints each (around 3 minutes per session), with approximately a one minute break in between each session where the participant could remove the virtual reality goggles and rest. These short breaks were enforced to reduce the probability of motion sickness due to the virtual reality goggles [27]. s either flew with haptic guidance $(\mathrm{N}=11)$ or without haptic guidance $(\mathrm{N}=9)$. The haptic guidance setup of the FlyJacket was the same as the one used in [3]. The haptic guidance corrected the user's torso position by pulling it toward a position that would allow them to fly a pre-calculated, optimal trajectory passing through the waypoints. The force rendered to the torso, $F_{\text {quad }}$ was quadratically proportional to the distance between the center of the waypoint and the drone, $\delta x(3)$.

$$
F_{\text {quad }}= \begin{cases}0.15 \cdot \delta x & |\delta x| \leq 14 \\ 30 & \text { otherwise }\end{cases}
$$

For small errors $(<4 \mathrm{~m})$, the haptic guidance gives a weak correction force $(<3 \mathrm{~N})$; therefore, the participant avoids being strongly perturbed. When the error becomes more significant, this guidance pulls the user strongly towards the reference trajectory. The maximum force given by the haptic guidance is $30 \mathrm{~N}$ per motor for an error larger than $14 \mathrm{~m}$. The same force command was given for the front and the back of the torso. After finishing these training tasks, both groups (with and without the haptic guidance) again filled out a questionnaire about their flight experience. The questionnaire was the same as the one given after the baseline task, but additional questions about the haptic guidance were given to the group that had received it. The additional statements were the following:

9) "Apart from the haptic feedback, my movements did not feel constrained during the flight,"

10) "The haptic feedback helped me to correct my trajectory,"

11) "The haptic feedback was on time with the flight."

In addition, they had to rate on a Likert scale from 1 (too weak) to 7 (too strong) the overall force intensity and for each body side (front, back, right, left). Participants also completed a NASA-TLX test, which measures the perceived amount of workload during this flight task [28].

The third and last task of this study was the evaluation task - a flight through 34 waypoints without haptic guidance for both groups. The aim of this part was to determine how well participants performed when the haptic guidance was removed in comparison to participants that did not receive any haptic guidance. At the end of this task, the participant had to fill the questionnaire about the flight sensations once more.

To score each flying task, the distance between the center of each waypoint and the point where the trajectory of the drone crosses a plane drawn perpendicular to the line connecting the previous and next waypoint was recorded (see Fig. 1D, for more details see [31]). This distance represents the error at each waypoint. The baseline score of each participant was calculated as the root mean square (RMS) of this error over the 26 waypoints of this task. The flight performance for the training and the evaluation task was evaluated as an error reduction relative to the performance achieved during the baseline task. This error reduction was computed by subtracting the error at each waypoint of the training task or the evaluation task from the RMS error of the baseline task. A larger error reduction means a greater improvement in performance. The data of the training and the evaluation tasks was fit with a linear curve (using the polyfit function in MATLAB) to determine the learning curve for these two tasks. The difference between the groups and their statistical significance were evaluated with a Wilcoxon Rank Sum test and one-sample t-test.

\subsection{Results of the Flight Experiment}

The error reduction during training and evaluation tasks was evaluated (Fig. 6) for each participants' flight through 150 waypoints (wp) during the training task and through $34 \mathrm{wp}$ for the evaluation task. The slope of the linear fitting of the data was similar for both group with $0.0040 \pm 0.0124$ $\mathrm{m} / \mathrm{wp}$ (mean $\pm \mathrm{SD}$ ) for the group flying with guidance and $0.0062 \pm 0.0221 \mathrm{~m} / \mathrm{wp}$ for the group flying without guidance $(P=0.970)$. Both groups show no statistically significant improvement over time ( $P=0.313$ for the group flying with haptic guidance and $P=0.428$ for the group flying without guidance). The performance improvement of the training task is immediate with an offset of the linear fitting of $5.43 \pm 6.40 \mathrm{~m}$ for the participants training with haptic feedback and $3.58 \pm 3.48 \mathrm{~m}$ for the participants training without haptic feedback. Both improvements are statistically significant $(P=0.0184$ and $P=0.0151$ respectively), but the difference between the offset of both groups was not significant $(P=0.880)$. 


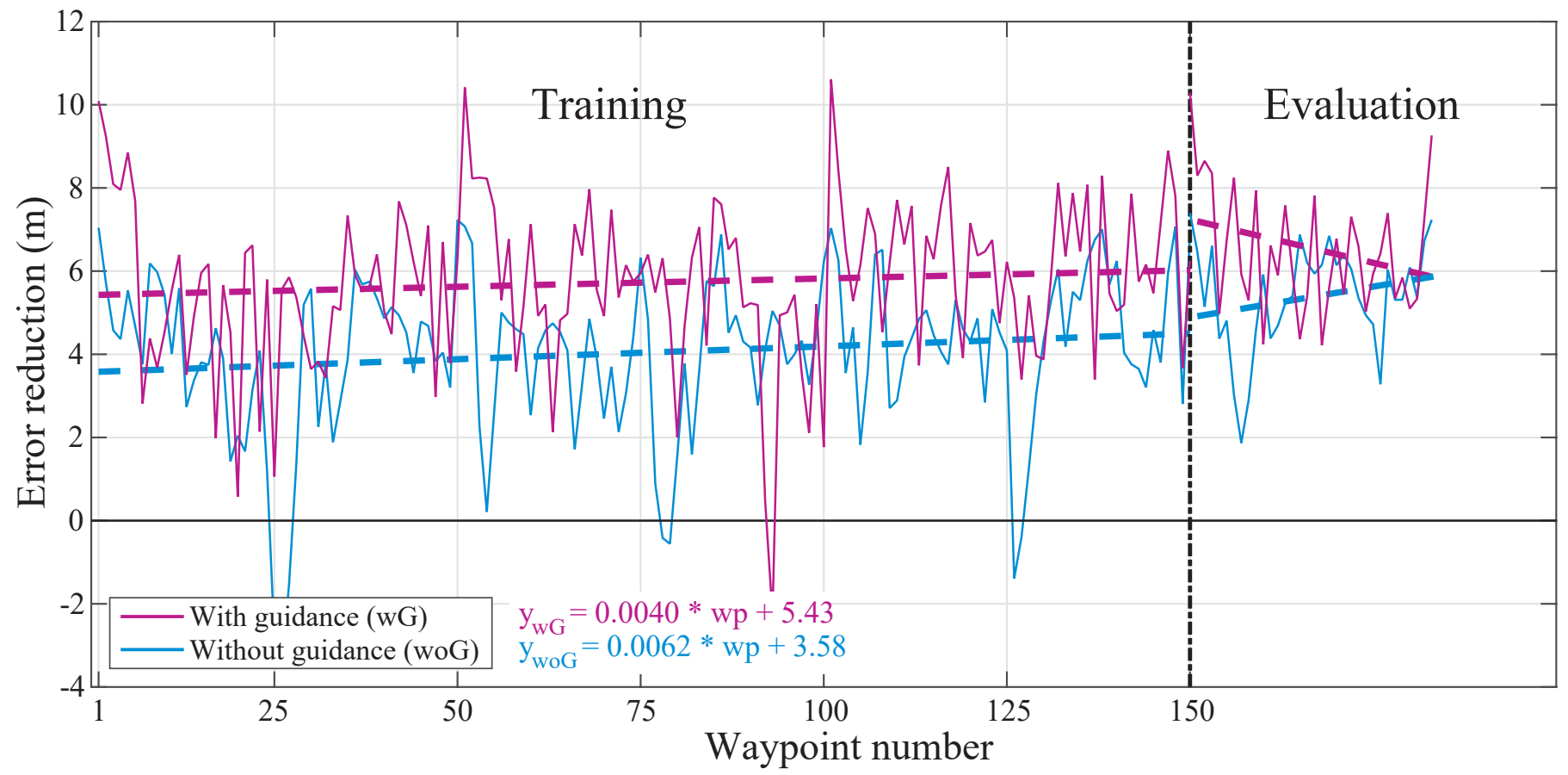

Fig. 6. The error reduction for both the training and evaluation flight tasks, relative to the RMS error of the baseline task, for participants that trained with guidance ( $\mathrm{N}=11$, magenta) and without guidance $(\mathrm{N}=9$, blue). Participants also had a five to seven minute break (dashed line) to fill a questionnaire between the end of the training task and the beginning of the evaluation task.

After the training, both groups completed an evaluation task without guidance to assess whether they retained the skills acquired during the training. The participants that trained without guidance continued to improve their performance at, surprisingly, a faster rate of $0.0296 \pm 0.0994$ $\mathrm{m} / \mathrm{wp}$ than during training. Comparatively, participants that trained with guidance started the evaluation task with a better error reduction on average than at the end of the training with an offset of error reduction of $7.24 \pm 6.48$ $\mathrm{m}$. They started also with a better mean performance than participants that trained without feedback $(4.89 \pm 6.08 \mathrm{~m})$. However, over time their performance degraded without haptic guidance with a rate of $-0.0416 \pm 0.0778 \mathrm{~m}$. The difference between the trend and offset for the two curves are not significant $(P=0.197$ for the slopes and $P=0.0965$ for the offsets) and their performance tended towards the same level of performance as the participants trained without guidance at the end of the evaluation task.

When flying with the haptic guidance, the same force was given to the front and back of the torso for the same level of error. The results of the experiment on the haptic perception described earlier on this article demonstrated a slightly lower perception of the magnitude of a given force applied to the back in comparison to the front. Therefore, we compared mean performance during training between "up" and "down" maneuvers (Fig. 7). For participants that trained with guidance, the error reduction was statistically higher $(P=0.049)$ for the "down" maneuvers $(7.45 \pm 2.30$ $\mathrm{m})$ - when the haptic guidance is pulling on the front of the torso - than for the "up" maneuvers $(4.51 \pm 3.62$ $\mathrm{m})$ - when the haptic guidance is pulling on the back of the torso - during the training task (Fig. 7). There is no significant difference in error reduction in any directions for

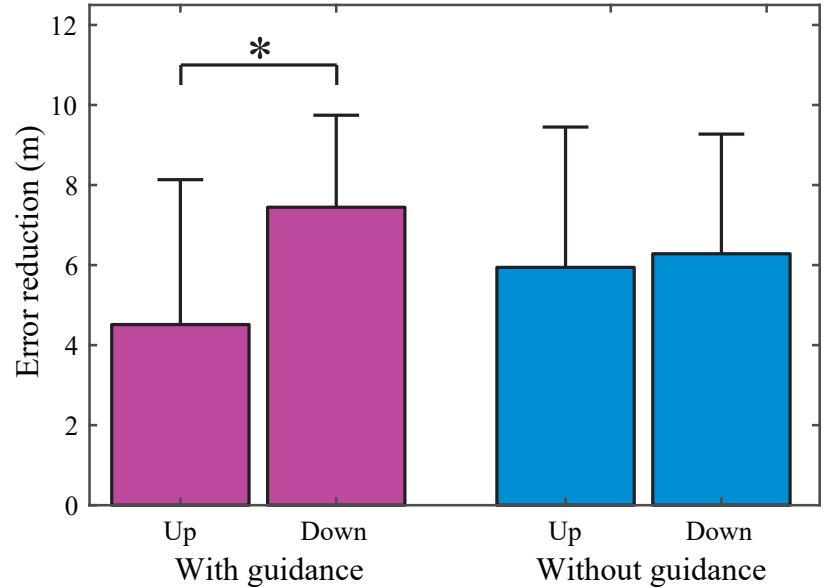

Fig. 7. Mean and standard deviation of the error reduction for "up" and "down" maneuvers during the training task for participants training with haptic guidance $(\mathrm{N}=11)$ and without haptic guidance $(\mathrm{N}=9)$. With haptic guidance, an "up" maneuvers corresponds to a pull on the back of the torso, and a "down" for a pull on the front of the torso. Asterisk $\left(^{*}\right)$ denotes $p<0.05$

the participants that trained without guidance (Fig. 7). This corroborates the results found with the haptic perception experiment that the participants feel the haptic feedback applied on the front of their torso with a higher magnitude than on the back.

The questionnaires completed by the participants after each task assessed their subjective feeling on their ability to control the trajectory and the comfort of the flight. No significant differences were found between the group training with guidance and the group without guidance. Adding haptic guidance to the flight did not physically constrain 
the user or prevent them from enjoying the flight tasks. There were also no significant differences in participant responses on the questionnaire's statements 1 to 8 among the baseline, training and evaluation tasks. From responses on the NASA-TLX questionnaire, there were no significant differences in workload between the group flying with the haptic guidance and the group flying without guidance with a workload of $54.96 \pm 19.19$ (on a scale from 1 to 100) for the people flying with feedback and $51.25 \pm 19.26$ for people flying without feedback. The haptic guidance does not alleviate or aggravate the subjective workload. There was no significant correlation between the physical characteristics (e.g. age, BMI, vision, handedness, gender, hours of physical activities per week) or their experience with VR goggles, piloting remotely an aircraft, or computer gaming of the participants and their performance of piloting the drone independently than if they receive an haptic guidance during their training or not.

\subsection{Discussion}

During a training period of approximately nine minutes, haptic guidance did not seem to help users learn the flying task faster than without guidance. However, haptic guidance improved performance instantaneously without increasing the workload on the user or degrading the sensation of flight. Therefore, flying with the haptic guidance could be a useful assistance mostly for naive users as they can immediately start flying with more precision. By averaging the performance of the participants, data show a trend of improvement over time with a better performance for the group training with the haptic feedback and also a better average performance during the evaluation. However, significant effects of the training over time was hard to assess because of the large variance of performance among participants. This large variance was present for both groups and is therefore independent of the haptic guidance.

One surprising behavior is the higher learning rate of the participant that was trained without the guidance during the evaluation task. One hypothesis is that they still had room for improvement and therefore continued to learn. Their faster learning rate, regarding to the training task, may be because flying during a long period is tiring and the five to seven minutes break between the training and the evaluation tasks allowed them to recover. A step in the error reduction is also seen between the baseline task and the training task, where participants had a five minutes break to fill the questionnaire. Apparently, a few minutes break may have influenced the error reduction during the next flight task and seemed to have a positive effect on the performance of both groups. However, the effect of these breaks is unknown, and specific experiments should be performed to understand precisely their influence on the learning process of participant, which is out of the scope of this article.

The perception of the haptic feedback had an influence on the error reduction. Indeed, from the perception experiments described earlier on this article, the minimum force difference is slightly but not significantly smaller for the front than for the back of the torso. Also, the magnitude of a given stimulus is perceived slightly larger on the front than on the back. These differences of perception were observed on the performance of the participants training with the haptic feedback as the error reduction was significantly higher when the haptic cue was given on the front of the torso than on the back. This difference was not observed in the group of participants flying without the haptic feedback. As apparently even non-significant variations on the perception lead to changes in performance in the flight task, quantification of the minimal force that the user can perceived is important. It was observed in the perception experiment that participants could not discern guidance forces below an error of $2.2 \mathrm{~m}$ (see section 2.4). Although it has been demonstrated in previous study that having a weak guidance force for small error prevents the user to be too frequently perturbed by the force and results in better performance than a hard guidance [3], possibly the force curve could be slightly tuned to still give light but perceivable guidance below this threshold.

Additional points should be considered to explain how participants had the same learning rate with and without guidance. The tuning of the guidance force was set on static perception experiment. Perhaps dynamic torso behavior somehow influenced force perception and should be taken into account in the force profile.

As the performance of the participants that trained with guidance decreases over time during the evaluation task, we hypothesis that participant got dependant on the feedback and relied on it to flight instead of learning. In addition, the results showed a large variation in performance between the participants. To tackle both of these issues, a possibility to improve the flight performance is to implement a personalized haptic guidance i.e. the stimulus intensity and frequency would be adapted to each user and could also adjust over time regarding the performance and confidence of the user in the flight. This implementation could improve the performance of individuals that were less responsive to the haptic guidance and reduce the variance of the overall performance. For each of these proposed approaches, experiments should be conducted to determine how they individually affect the learning rate and skill retention over an extended period of time.

\section{Conclusion}

The work presented in this paper shows that a cable-driven force feedback to the human torso is perceived linearly with the applied stimulus. These results corroborate with previous experiments on the perception of vibrotactile feedback [22]. The force perception was observed to be slightly lower on the back of the torso, both during the perception studies as well as during the flight experiments. This imbalance can have repercussions in the flight performance and can be addressed by appropriately modulating the magnitude of the applied force with larger control gains for the back motors. We also demonstrated that a guidance-based haptic feedback provided through the FlyJacket boosts user performance during the training phase of a path-following task. However, this improvement in performance was not significantly greater than training without feedback, and these gains might subsequently be lost when this feedback is taken away. This could be indicative of poor skill retention, 
possibly due to users developing a dependence on the guidance or "slacking" [29]. More thorough retention tests need to be conducted to assess and distinguish the shortterm and long-term effects of the flight training.

In this work, one method of haptic feedback guidance was explored to understand its effect on user performance, motor learning, and skill retention. The perception test performed investigated stimuli in static positions. As a followup, we are currently carrying out a participant study to examine the dynamic response of a human torso when it is subjected to a continuous sinusoidal kinesthetic stimulus. The stimulus is provided to the torso via the same motorized cable driven system used in the perception and motor learning studies shown in this paper. More specifically, we are interested in establishing relationships among torso movement, the applied stimulus frequency, the location of the cable attachment points on the torso, and intersubject anthropometry. Furthermore, we are developing a neuromechanical model to capture the dynamical torso response to input stimulus and quantify cognitive alertness and mechanical passivity as model parameters for each participant. Separately, we will be investigating dynamic torso perceptiveness i.e., the dependence of threshold perceptible force on velocity for $\mathrm{s}$ in motion. Our aim is to use the model to predict the response of each individual in a flight teleoperation task and the dynamic perception tests to ascertain the type of feedback that should be provided to participants to augment the rate of their motor skill acquisition.

In the future, the FlyJacket will be used to test a combination of different approaches to target improved human robot interaction. Indeed, one possibility is to use the same hardware setup for haptic feedback provision but instead of guiding users, the cable-driven feedback could be used to hinder users from achieving their target. This approach would draw on some exhibited benefits of error amplification as a pedagogical tool in haptics studies [30]. Still using the same hardware setup, guidance can be given to prevent the drone from colliding with an obstacle or from performing dangerous maneuvers that are outside the flight envelope, such as flying at a too high angle of attack. In these critical conditions, the movements of the user can be restricted using this type of haptic guidance.

Alternatively, one could employ passive haptic feedback, a type of feedback that combines certain favourable attributes of haptic guidance and error amplification to hinder erroneous joint movement. Here, a system of brakes and clutches can be embedded into the existing FlyJacket to have a more body-conforming design [31] [32]. With passive haptic feedback, users would be given more agency to make mistakes, identify those mistakes with the feedback provided, and correct for them.

As another approach to improve the flight performance, tactile feedback to render the sensation of flight can be embedded into the existing FlyJacket. A system of closed air pouches which produces the sensation of air being compressed against the torso during drone's centripetal acceleration has been developed and tested [?].

Using the FlyJacket to control a drone is one form of teleoperation. The cable-driven haptic guidance in the form of kinesthetic feedback studied is this article can be used in other types of teleoperation, such as the balance of a bipedal humanoid robot like the Hermes robot [33] or, if placed on the users arm, kinesthetic feedback during teleoperation of a robotic arm.

\section{ACKNOWLEDGMENTS}

This work was supported by the Swiss National Science Foundation through the National Centre of Competence in Research Robotics (NCCR Robotics) and through the FLAGERA project RoboCom++.

\section{REFERENCES}

[1] D. Floreano and R. J. Wood, "Science, technology and the future of small autonomous drones," Nature, vol. 521, no. 7553, p. 460, 2015.

[2] J. A. Adams, "Critical considerations for human-robot interface development," in Proceedings of 2002 AAAI Fall Symposium, 2002, pp. 1-8.

[3] C. Rognon, A. R. Wu, S. Mintchev, A. Ijspeert, and D. Floreano, "Haptic guidance with a soft exoskeleton reduces error in drone teleoperation," in International Conference on Human Haptic Sensing and Touch Enabled Computer Applications. Springer, 2018, pp. 404415.

[4] T. M. Lam, M. Mulder, and M. R. van Paassen, "Haptic interface in uav tele-operation using force-stiffness feedback," in Systems, Man and Cybernetics, 2009. SMC 2009. IEEE International Conference on. IEEE, 2009, pp. 835-840.

[5] M. Olivari, F. M. Nieuwenhuizen, H. H. Bülthoff, and L. Pollini, "Pilot adaptation to different classes of haptic aids in tracking tasks," Journal of Guidance, Control, and Dynamics, vol. 37, no. 6, pp. 1741-1753, 2014.

[6] X. Hou, R. E. Mahony, and F. Schill, "Comparative study of haptic interfaces for bilateral teleoperation of vtol aerial robots." IEEE Trans. Systems, Man, and Cybernetics: Systems, vol. 46, no. 10, pp. 1352-1363, 2016

[7] A. Kanso, I. H. Elhajj, E. Shammas, and D. Asmar, "Enhanced teleoperation of uavs with haptic feedback," in Advanced Intelligent Mechatronics (AIM), 2015 IEEE International Conference on. IEEE 2015, pp. 305-310.

[8] J. Miehlbradt, A. Cherpillod, S. Mintchev, M. Coscia, F. Artoni, D. Floreano, and S. Micera, "Data-driven body-machine interface for the accurate control of drones," Proceedings of the National Academy of Sciences, p. 201718648, 2018.

[9] C. Rognon, S. Mintchev, F. Dell'Agnola, A. Cherpillod, D. Atienza, and D. Floreano, "Flyjacket: An upper body soft exoskeleton for immersive drone control," IEEE Robotics and Automation Letters, vol. 3, no. 3, pp. 2362-2369, 2018.

[10] A. E. Sklar and N. B. Sarter, "Good vibrations: Tactile feedback in support of attention allocation and human-automation coordination in event-driven domains," Human factors, vol. 41, no. 4, pp. 543-552, 1999.

[11] J. L. Burke, M. S. Prewett, A. A. Gray, L. Yang, F. R. Stilson, M. D. Coovert, L. R. Elliot, and E. Redden, "Comparing the effects of visual-auditory and visual-tactile feedback on user performance: a meta-analysis," in Proceedings of the 8th international conference on Multimodal interfaces. ACM, 2006, pp. 108-117.

[12] M. M. Coad, A. M. Okamura, S. Wren, Y. Mintz, T. S. Lendvay, A. M. Jarc, and I. Nisky, "Training in divergent and convergent force fields during 6-dof teleoperation with a robot-assisted surgical system," in World Haptics Conference (WHC), 2017 IEEE. IEEE, 2017, pp. 195-200.

[13] T. Nef, M. Mihelj, and R. Riener, "Armin: a robot for patientcooperative arm therapy," Medical $\mathcal{E}$ biological engineering $\mathcal{E}$ computing, vol. 45, no. 9, pp. 887-900, 2007.

[14] G. Rauter, R. Sigrist, R. Riener, and P. Wolf, "Learning of temporal and spatial movement aspects: A comparison of four types of haptic control and concurrent visual feedback," IEEE transactions on haptics, vol. 8, no. 4, pp. 421-433, 2015.

[15] R. Sigrist, G. Rauter, R. Riener, and P. Wolf, "Augmented visual, auditory, haptic, and multimodal feedback in motor learning: a review," Psychonomic bulletin \& review, vol. 20, no. 1, pp. 21-53, 2013. 
[16] J. D. Brown, M. K. Shelley, D. Gardner, E. A. Gansallo, and R. B. Gillespie, "Non-colocated kinesthetic display limits compliance discrimination in the absence of terminal force cues," IEEE transactions on haptics, vol. 9, no. 3, pp. 387-396, 2016.

[17] M. H. Zadeh, D. Wang, and E. Kubica, "The effect of sub-threshold forces on human performance in multi-modal computer-aided design," Computer-Aided Design, vol. 42, no. 5, pp. 471-477, 2010.

[18] J. M. Wolfe, K. R. Kluender, D. M. Levi, L. M. Bartoshuk, R. S. Herz, R. L. Klatzky, S. J. Lederman, and D. M. Merfeld, Sensation E perception. Sinauer Sunderland, MA, 2006.

[19] J. C. Baird and E. J. Noma, Fundamentals of scaling and psychophysics. John Wiley \& Sons, 1978.

[20] S. S. Stevens, "On the psychophysical law," Psychological review, vol. 64, no. 3, p. 153, 1957.

[21] — - Psychophysics: Introduction to its perceptual, neural and social prospects. Routledge, 2017.

[22] _ "To honor fechner and repeal his law," Science, vol. 133, no. 3446, pp. 80-86, 1961.

[23] T. N. Cornsweet, "The staircase-method in psychophysics," The American journal of psychology, vol. 75, no. 3, pp. 485-491, 1962.

[24] J. K. Holden, E. M. Francisco, Z. Zhang, C. Baric, and M. Tommerdahl, "An undergraduate laboratory exercise to study webers law," Journal of Undergraduate Neuroscience Education, vol. 9, no. 2, p. A71, 2011.

[25] E. Francisco, V. Tannan, Z. Zhang, J. Holden, and M. Tommerdahl, "Vibrotactile amplitude discrimination capacity parallels magnitude changes in somatosensory cortex and follows webers law," Experimental brain research, vol. 191, no. 1, p. 49, 2008.

[26] S. Weinstein, "Intensive and extensive aspects of tactile sensitivity as a function of body part, sex and laterality," The skin senses, 1968.

[27] J. J. LaViola Jr, "A discussion of cybersickness in virtual environments," ACM SIGCHI Bulletin, vol. 32, no. 1, pp. 47-56, 2000.

[28] S. G. Hart and L. E. Staveland, "Development of nasa-tlx (task load index): Results of empirical and theoretical research," in Advances in psychology. Elsevier, 1988, vol. 52, pp. 139-183.

[29] L. Marchal-Crespo and D. J. Reinkensmeyer, "Review of control strategies for robotic movement training after neurologic injury," Journal of NeuroEngineering and Rehabilitation, vol. 6, no. 1, p. 20, Jun. 2009. [Online]. Available: https://doi.org/10.1186/17430003-6-20

[30] M.-H. Milot, L. Marchal-Crespo, C. S. Green, S. C. Cramer, and D. J. Reinkensmeyer, "Comparison of error-amplification and hapticguidance training techniques for learning of a timing-based motor task by healthy individuals," Experimental brain research, vol. 201, no. 2, pp. 119-131, 2010.

[31] V. Ramachandran, J. Shintake, and D. Floreano, "All-fabric wearable electroadhesive clutch," Advanced Materials Technologies, p. 1800313, 2018.

[32] A. Tonazzini, J. Shintake, C. Rognon, V. Ramachandran, S. Mintchev, and D. Floreano, "Variable stiffness strip with strain sensing for wearable robotics," in 2018 IEEE International Conference on Soft Robotics (RoboSoft). IEEE, 2018, pp. 485-490.

[33] A. Wang, J. Ramos, J. Mayo, W. Ubellacker, J. Cheung, and S. Kim, "The hermes humanoid system: A platform for full-body teleoperation with balance feedback," in 2015 IEEE-RAS 15th International Conference on Humanoid Robots (Humanoids). IEEE, 2015, pp. 730737.

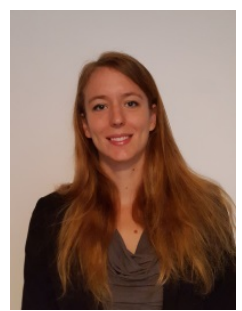

Carine Rognon received a M.Sc. in Biomedical Engineering from the Eidgenssische Technische Hochschule Zurich (ETHZ), Switzerland, in 2015, and a B.Sc in Microengineering from the Ecole Polytechnique Fédérale de Lausanne (EPFL), Switzerland, in 2013. She is currently a PhD student in robotics and member of the Swiss National Center of Competence in Robotics (NCCR Robotics). Her research interests include human machine interaction, haptics and human perception, and soft exoskeletons.

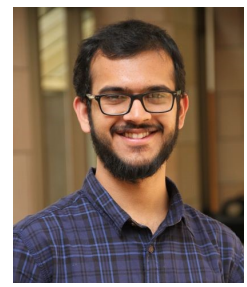

Vivek Ramachandran is a PhD student at the Laboratory of Intelligent Systems at the École Polytechnique Fédérale de Lausanne (EPFL), Switzerland. He received his M.S. from Carnegie Mellon University in 2016 and B.tech from the National Institute of Technology Calicut in 2014, both in Mechanical Engineering. He is currently a $\mathrm{PhD}$ student in robotics and member of the Swiss National Center of Competence in Robotics (NCCR Robotics). His research interests include mechanics of materials, soft matter engineering, haptics, and wearable robotics.

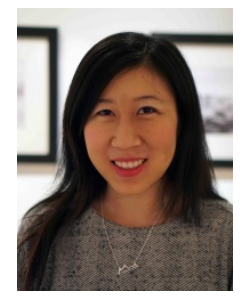

Amy $\mathbf{R}$. Wu is a postdoctoral researcher in the Biorobotics Laboratory at the Ecole Polytechnique Fédérale de Lausanne (EPFL), Switzerland. She completed her Ph.D. in Mechanical Engineering in the Human Biomechanics and Control Lab at the University of Michigan. She received her S.B. from the Massachusetts Institute of Technology and her M.S.E. from the University of Michigan, Ann Arbor, both in Aerospace Engineering. Her research interests locomotion. are in biomechanics and control of balance and

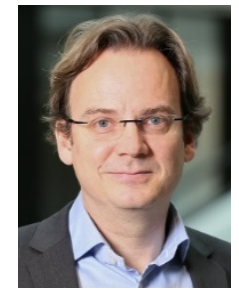

Auke ljspeert is a full professor at the Ecole Polytechnique Fédérale de Lausanne (EPFL), Switzerland, and head of the Biorobotics Laboratory (BioRob). He has a B.Sc./M.Sc. in physics from the EPFL (1995) and a PhD in artificial intelligence from the University of Edinburgh (1999). He carried out postdocs at IDSIA and EPFL, and at the University of Southern California (USC). His primary affiliation is with the Institute of Bioengineering, and secondary affiliation with the Institute of Mechanical Engineering. His research interests are at the intersection between robotics, computational neuroscience, nonlinear dynamical systems, and applied machine learning.

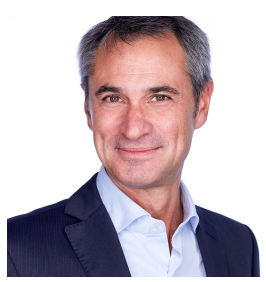

Dario Floreano is Director of the Laboratory of Intelligent Systems at EPFL Switzerland and Director of the Swiss National Center of Robotics. $\mathrm{He}$ has an M.Sc. in neural computation from the University of Stirling in 1992, and a Ph.D. in cognitive systems and robotics from the University of Trieste in 1995 . His research focuses on the convergence of biology, artificial intelligence, and robotics. He has published more than 300 peer-reviewed papers and four books on the topics of evolutionary robotics, bio-inspired artificial intelligence, and bio-mimetic flying robots with MIT Press and Springer Verlag. 